https://communications.science.ankara.edu.tr

Commun.Fac.Sci.Univ.Ank.Series A2-A3

Volume 63, Number 1, Pages 58-79 (2021)

DOI: 10.33769 /aupse. 851493

ISSN 1303-6009 E-ISSN 2618-6462

Received by the Editors: January 17, 2021; Accepted: April 05, 2021

\title{
A NEW ASTRONOMICAL PARAMETER FROM REMOTE SENSING DATA: ASTRONOMICAL CLEARNESS INDEX (ACI)
}

\author{
Kazım KABA $^{1}$, Cahit YEŞILILYAPRAK ${ }^{1,2}$ and Onur ŞATIR ${ }^{1}$ \\ ${ }^{1}$ Astrophysics Research and Application Centre (ATASAM), Atatürk University, \\ Erzurum, TURKEY \\ ${ }^{2}$ Department of Astronomy and Space Sciences, Science Faculty, Atatürk University, \\ Erzurum, TURKEY
}

\begin{abstract}
Eastern Anatolia Observatory (DAG) project was initiated in Erzurum/Turkey in 2011. DAG will have Turkey's largest $(4 \mathrm{~m})$ and first infrared telescope. The installation process is planned to be by taking its first light in the end of 2021. This study was focused on a new analysis method about the atmospheric properties of DAG site in terms of the cloudiness as known the most vital atmospheric parameter for ground-based astronomical observatories. In this regard, the cloudiness for DAG site is comprehensively examined using the "Cloud Mask" (CMa) and "Cloud Type" (CT) products from Satellite Application Facility on Support to Nowcasting and Very Short-Range Forecasting (NWC SAF). Firstly, the cloudiness and the cloud types over DAG site were determined. Secondly, NWC SAF CMa and CT data have been redefined for astronomical purposes, and the pixel values/meanings in CMa and CT images have been reduced from 6 to 4 and from 21 to 4 pixels, respectively. Thirdly, these new data were used to define a new index named as "Astronomical Clearness Index" (ACI), and finally, the observable days for DAG site were determined using this newly defined index.
\end{abstract}

\section{INTRODUCTION}

One of the most determining factors for ground-based astronomical observations is the atmosphere of the Earth. Continuous monitoring of the characteristic features of the atmosphere with high accuracy is crucial to plan and prepare observation types, projects and devices, and of course to obtain quality observational data. This issue is also important for the effective use of valuable observation time for an observatory. The quality of the Earth's atmosphere for astronomical observation is evaluated by

Keywords and phrases. Astronomical clearness index, astronomy, cloud mask and types, Eastern Anatolia Observatory, remote sensing

kkaba46@gmail.com-Corresponding author; cahity@atauni.edu.tr; onur.satir@gmail.com

0000-0001-8328-8123; 0000-0003-1894-7190; 0000-0002-6161-8627 
primary parameters such as cloudiness, temperature, precipitation, pressure, humidity and wind which are fundamental for the meteorological studies. The first main meteorological parameter for all astronomical observations (visible or infrared) is the cloudiness information (e.g. clouds coverage, cloud types, cloud coordinates on the sky, cloud altitude, or time-dependent cloud changes). The cloudiness information for an observatory site not only gives information about the quality of the site chosen for the observatory and how often it is suitable for observation, but also provides a great opportunity to prepare the observational devices to be used and decide on the types of observations.

In astronomy, meteorological data are widely used for an observatory site selection, and instant and continuous monitoring of short-term and long-term atmospheric conditions. Meteorological analyses are also important for scheduling the observations during time allocation for observations, especially. There are many studies conducted in this context and cloudiness information is evaluated especially in order to determine the number of the "clear nights" to observe [1-10].

Today, meteorology obtains the cloudiness information needed by different disciplines (agriculture, transportation, astronomy etc.) in different ways. Traditionally, the cloudiness information is determined as a percentage by the observers at meteorological stations; in recent years, it can be obtained by analysing the images of the sky taken with the ground-based wide-angle cameras (ASC: All Sky Camera). Also, numerical weather forecast models produce cloudiness information. Another way to obtain the cloudiness information is to use remote sensing methods from some meteorological satellites in certain orbits around the Earth. From the satellite data, cloud mask (CMa), cloud type (CT) and cloud fraction (CF) information are obtained as categorical and continuous values of cloudiness information. Cloud data is used in studies of different disciplines such as energy, transportation, climate change and astronomy [9, 11-13].

Nowadays, for astronomical observatories, the instant and continuous monitoring of the atmospheric and astronomical conditions, and predictions have been made with much more accuracy by remote sensing methods and the analysis of meteorological satellite data. Such studies have become indispensable today for all observatories having a large telescope. Remote sensing data has many advantages over groundbased data such as being digital, covering large areas and low costs.

In this study, the geostationary meteorological satellite (METEOSAT) data, which covers Turkey as well, was used to determine the method for cloudiness. The NWC SAF (Satellite Application Facility for Nowcasting and Very Short-Range 
Forecasting) unit of EUMETSAT (European Organisation for the Exploitation of Meteorological Satellites) produces various data sets from METEOSAT data for short-term weather forecast. NWC SAF cloud products are generated from records of the SEVIRI (Spinning Enhanced Visible and InfraRed Imager) sensor onboard METEOSAT satellites. We used two of these data sets together (CMa and CT products) for the first time, to define an astronomical cloudiness parameter (ACI: Astronomical Clearness Index). Using this new index (ACI) together with the ASC data obtained from the Eastern Anatolian Observatory (DAG) site $(3170 \mathrm{~m}$, Karakaya summit, Konakl1, Erzurum), the cloudiness information and characteristics of DAG site were determined for the first time.

\section{Site, Data And Method}

\subsection{Eastern Anatolia Observatory (DAG) Site}

Eastern Anatolia Observatory (DAG) is located at the coordinates of 39.78 degree of North latitude and 41.23 degree of East longitude on the Karakaya summit with an altitude of $3170 \mathrm{~m}$, within the 2500 acres of land, in Erzurum, Turkey. Erzurum, which is a city in the east part of Turkey, and DAG site can be seen in Figure 1.

The basic climatic characteristics for Erzurum are of a dominantly continental climate; winters are long, cold and snowy; summers are short and warm. The basic meteorological properties for Erzurum city and DAG site are given in Table 1. The data for Erzurum were taken from the web page ${ }^{1}$ of TSMS (Turkish State Meteorological Service).

\footnotetext{
${ }^{1}$ https://www.mgm.gov.tr/veridegerlendirme/il-ve-ilceler-istatistik.aspx?k=undefined $\backslash \& \mathrm{~m}=$ ERZURUM
} 


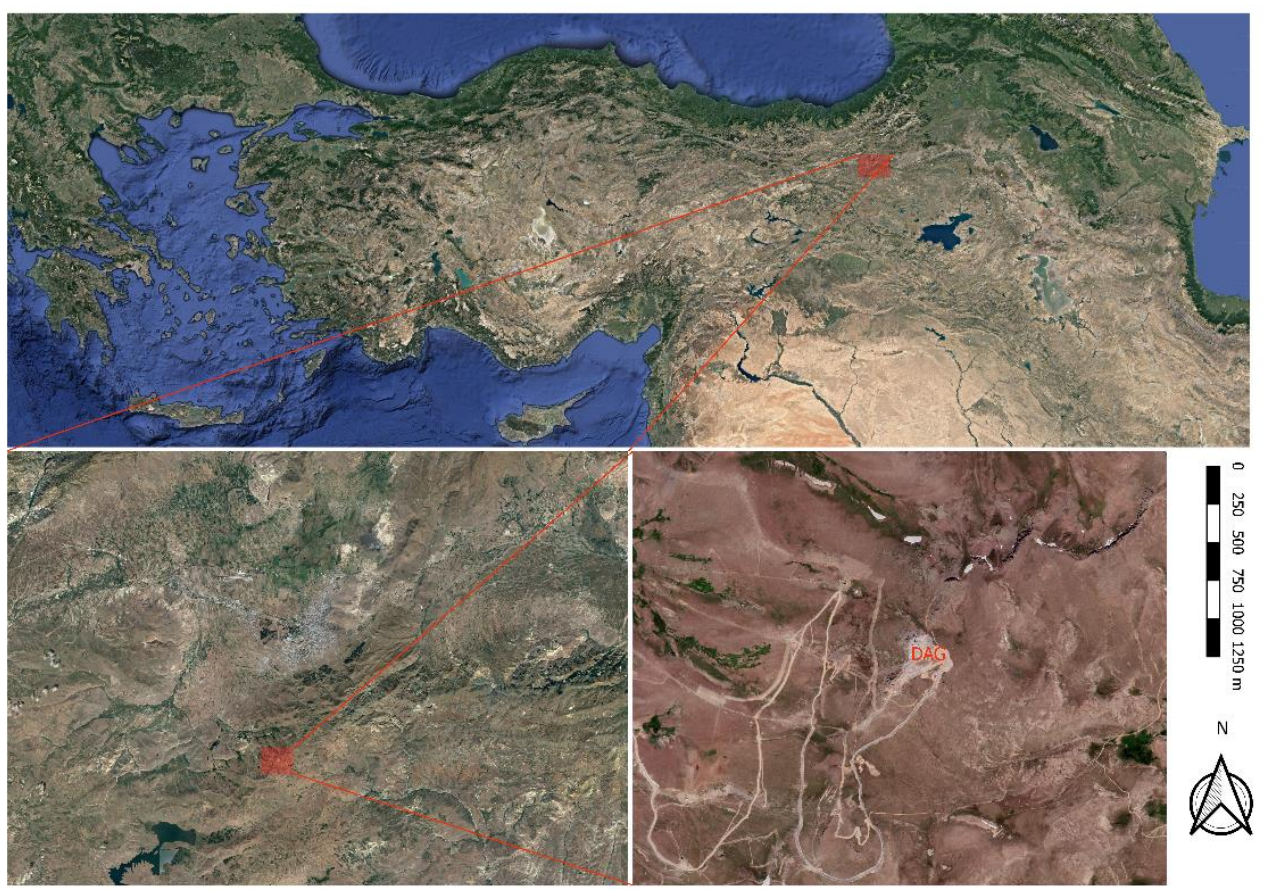

Figure 1. DAG Site at $3170 \mathrm{~m}$ altitude, Karakaya summit in Konakl, Erzurum, Turkey.

TABLE 1. The basic meteorological properties for Erzurum city and DAG site. The measurements are taken from TSMS and DAG databases, and recorded between 1929 - 2019, and 2012 - 2019 for Erzurum and DAG site, respectively.

\begin{tabular}{|c|c|c|c|c|c|c|c|}
\hline Location & $\begin{array}{c}\text { Altitude } \\
(\mathbf{m})\end{array}$ & $\begin{array}{c}\text { T } \\
\text { Winter } \\
\left({ }^{\circ} \mathbf{C}\right)\end{array}$ & $\begin{array}{c}\mathbf{T}_{\text {mean }} \\
\text { Summer } \\
\left({ }^{\circ} \mathbf{C}\right)\end{array}$ & $\begin{array}{c}\mathbf{T}_{\min } \\
\left({ }^{\circ} \mathbf{C}\right)\end{array}$ & $\begin{array}{c}\mathbf{T}_{\max } \\
\left({ }^{\circ} \mathbf{C}\right)\end{array}$ & $\begin{array}{c}\mathbf{H}_{\text {mean }} \\
(\mathbf{\%})\end{array}$ & $\begin{array}{c}\mathbf{V}_{\text {mean }} \\
(\mathbf{m} / \mathbf{s})\end{array}$ \\
\hline Erzurum & 1860 & -7.5 & 17.8 & -37.2 & 36.5 & $63.3^{*}$ & $2.7^{*}$ \\
\hline DAG site & 3170 & -9.1 & 12.4 & -34.5 & 32.8 & 54 & 6.9 \\
\hline
\end{tabular}




\subsection{Data}

\subsubsection{MSG-SEVIRI Instrument Characteristics}

SEVIRI (Spinning Enhanced Visible and InfraRed Imager), which is the primary sensor carried by MSG (Meteosat Second Generation) satellites, has 12 channels, and it provides images with $3 \mathrm{~km}$ spatial resolution (1 km for HRV: High Resolution Visible channel) and $15 \mathrm{~min}$ temporal resolution at full scan (5 min in fast scan mode). SEVIRI has four channels called visible or shortwave and eight channels called thermal (IR). SEVIRI's VIS0.6, VIS0.8, NIR1.6 and HRV channels are sensitive to Solar radiation reflected from the ground and the atmosphere. The best performing channel for detecting clouds is VIS0.6, for terrestrial properties and vegetation is VIS0.8, for ice and snow is NIR1.6, and for low clouds and fog is IR3.9. As the IR3.9 channel is permeable to radiation with wavelength centred at $3.9 \mu \mathrm{m}$, it is sensitive to Solar radiation reflected during the daytime, and thermal radiation emitted during an entire day. The IR3.9 channel is primarily used for the detection of low clouds and fog, and wind vectors. This channel also supports ground and water surface temperature measurements for night time. Since the IR3.9 channel is sensitive to $\mathrm{CO}_{2}$ absorption around $3.9 \mu \mathrm{m}, \mathrm{CO}_{2}$ correction is applied for the reflectance calculated from daytime records. HRV channel is a high resolution (1 $\mathrm{km})$ visible channel. By providing high-resolution images, this channel allows the processing of data from other channels to be more advanced. It can be used to check the presence of the clouds in the corresponding pixel on lower resolution channels of the displayed region. Therefore, it allows for more advanced cloud detection and sub-pixel processing of SEVIRI data for some products. The main features of the channels of the MSG SEVIRI sensor were given in Table 2.

Infrared channels are sensitive to thermal radiation emitted from land or ocean surfaces and clouds, and they provide information both day and night. The WV6.2 and WV7.3 channels are centred on $6.2 \mu \mathrm{m}$ and $7.3 \mu \mathrm{m}$, respectively, and sensitive to radiation emitted by the water vapor in the upper parts of the troposphere. These channels show the vortex-shaped moisture at a height of $5-8 \mathrm{~km}$ in the high-levelcloud-free parts of the area being viewed. While the IR8.7 channel is permeable to infrared radiation coming from the window centred around $8.7 \mu \mathrm{m}$, the IR9.7 channel is permeable to radiation emitted from $\mathrm{O}_{3}$ molecules at $9.7 \mu \mathrm{m}$. The IR10.8 and IR12.0 channels are centred at $10.8 \mu \mathrm{m}$ and $12.0 \mu \mathrm{m}$, respectively, and are channels showing clouds and surfaces at these wavelengths. The IR13.4 channel is permeable to radiation emitted from $\mathrm{CO}_{2}$ molecules at $13.4 \mu \mathrm{m}$.

The IR8.7 channel provides the separation of ice and water clouds, and information about the quantity of thin cirrus clouds. The IR10.8 and IR12.0 channels basically 
measure land and sea surface temperature, and cloud-top temperature. In addition, cirrus clouds and volcanic ash clouds are detected in these channels. The IR9.7 and IR13.4 channels are permeable to radiation emitted from clouds and surfaces as well as $\mathrm{O}_{3}$ and $\mathrm{CO}_{2}$ absorption. This effect is eliminated by comparison with other channels. Other channels are used to identify clouds and remove background surface radiation. When this is done, the remaining signal gives information about special atmospheric conditions $\left(\mathrm{O}_{3}\right.$ and $\left.\mathrm{CO}_{2}\right)$. While the IR9.7 channel is sensitive to the $\mathrm{O}_{3}$ density in the lower stratosphere, the IR13.4 channel is sensitive to $\mathrm{CO}_{2}$ gas at the bottom of the troposphere.

TABLE 2. The properties of MSG SEVIRI channels ${ }^{2}$.

\begin{tabular}{|c|c|c|c|c|c|}
\hline \multirow[b]{2}{*}{$\begin{array}{l}\text { Channel } \\
\text { Number }\end{array}$} & \multirow{2}{*}{$\begin{array}{c}\text { Spectral } \\
\text { Band } \\
(\mu \mathrm{m})\end{array}$} & \multicolumn{3}{|c|}{ Wavelength $(\mu \mathrm{m})$} & \multirow{2}{*}{$\begin{array}{l}\text { Main Observational } \\
\text { Application }\end{array}$} \\
\hline & & $\begin{array}{c}\lambda_{\min } \\
(\mu \mathrm{m})\end{array}$ & $\begin{array}{l}\lambda_{\text {center }} \\
(\boldsymbol{\mu} \mathrm{m})\end{array}$ & $\begin{array}{l}\lambda_{\max } \\
(\mu \mathrm{m})\end{array}$ & \\
\hline 1 & VIS0.6 & 0.56 & 0.635 & 0.71 & Surface, clouds, wind fields \\
\hline 2 & VIS0.8 & 0.74 & 0.81 & 0.88 & Surface, clouds, wind fields \\
\hline 3 & NIR1.6 & 1.50 & 1.64 & 1.78 & Surface, cloud phase \\
\hline 4 & IR3.9 & 3.48 & 3.90 & 4.36 & Surface, clouds, wind fields \\
\hline 5 & WV6.2 & 5.35 & 6.25 & 7.15 & $\begin{array}{c}\text { Water vapor, high level } \\
\text { clouds, atmospheric instability }\end{array}$ \\
\hline 6 & WV7.3 & 6.85 & 7.35 & 7.85 & $\begin{array}{c}\text { Water vapor, atmospheric } \\
\text { instability }\end{array}$ \\
\hline 7 & IR8.7 & 8.30 & 8.70 & 9.10 & $\begin{array}{c}\text { Surface, clouds, atmospheric } \\
\text { instability }\end{array}$ \\
\hline 8 & IR9.7 & 9.38 & 9.66 & 9.94 & Ozone \\
\hline 9 & IR10.8 & 9.80 & 10.80 & 11.80 & $\begin{array}{c}\text { Surface, clouds, wind fields, } \\
\text { atmospheric instability }\end{array}$ \\
\hline 10 & IR12.0 & 11.00 & 12.00 & 13.00 & $\begin{array}{l}\text { Surface, clouds, atmospheric } \\
\text { instability }\end{array}$ \\
\hline 11 & IR13.4 & 12.40 & 13.40 & 14.40 & $\begin{array}{l}\text { Cirrus cloud height, } \\
\text { atmospheric instability }\end{array}$ \\
\hline 12 & HRV & \multicolumn{3}{|c|}{ Broadband $(\sim 0.4-1.1 \mu \mathrm{m})$} & Surface, clouds \\
\hline
\end{tabular}

\subsubsection{NWC SAF Cloud Mask (CMa) Product}

The CMa product ${ }^{3}$ developed by the NWC SAF, aims to support nowcasting applications and to detect land-ocean surfaces remotely. The CMa product allows us to identify cloudless areas where other satellite products (such as precipitable water

${ }^{2}$ https://www.mgm.gov.tr/genel/meteorolojikuydular.aspx?s=61

${ }^{3}$ https://www.nwcsaf.org/cma_description 
vapor - PWV, land or sea surface temperature, snow or ice identification) can be calculated. It also enables the detection of cloudy areas where products such as CT and cloud-top temperature/height can be obtained. In addition, the product provides information about the presence of snow or sea ice, dust clouds, and volcanic ash (plumes) [15]. However, the main purpose of the CMa product is to give all cloudless pixels in a satellite image with high accuracy. Table 3 shows the pixel values and what they mean in CMa images produced with NWC SAF 2013 version. A sample CMa image produced with the 2013 version of NWC SAF is given in Figure 2. The sample CMa image is an instantaneous data and was obtained at 2020-01-01 00:00 UTC.

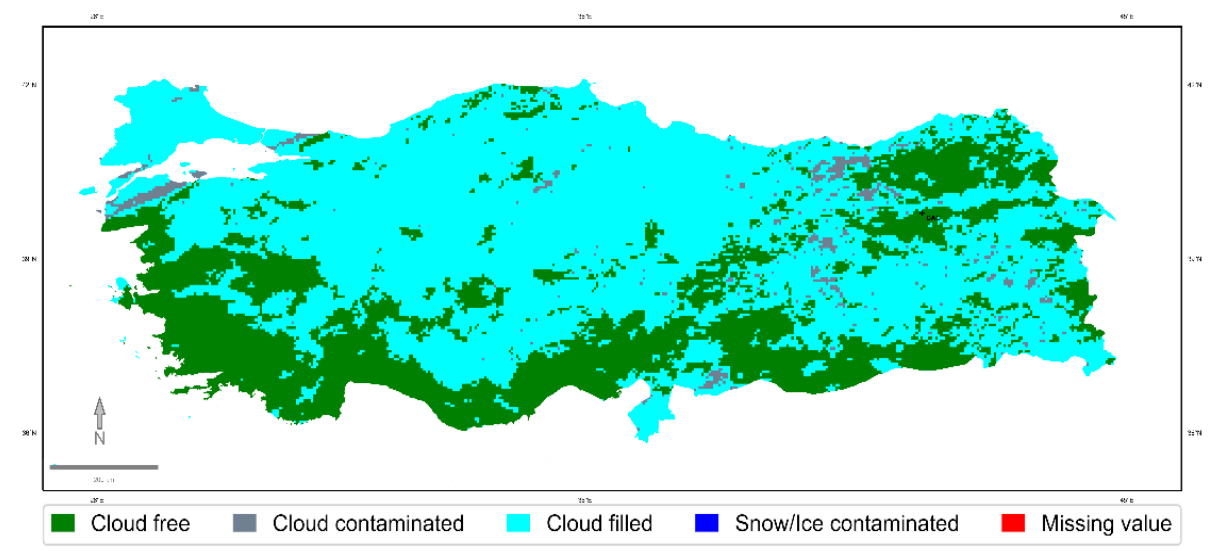

FIgURE 2. A Sample image that we produced using NWC SAF CMa data.

TABLE 3. Meaning of pixel values in NWC SAF CMa images.

\begin{tabular}{|c|c|}
\hline Pixel Value & CMa (Cloud Mask) \\
\hline 0 & Non-processed \\
\hline 1 & Cloud free \\
\hline 2 & Cloud contaminated \\
\hline 3 & Cloud filled \\
\hline 4 & Snow/Ice contaminated \\
\hline 5 & Undefined \\
\hline
\end{tabular}




\subsubsection{NWC SAF Cloud Type (CT) Product}

The CT product ${ }^{4}$, developed by the NWC SAF unit mainly aims to support nowcasting applications by providing a detailed cloud analysis. The CT product can be used as input for a mid-range target analysis, as an intermediate product input to other products or as a final image for a forecaster. For example, CT product is required to produce cloud-top temperature and cloud-top height products, and determination of precipitation clouds. In addition, this product is used to calculate the radiation flux on the sea or land. When a pixel defined as cloudy in a CMa images, the corresponding pixel in CT images contains information about main cloud classes such as partial or fractional clouds, semi-transparent clouds, high, medium and low clouds [15]. Table 4 shows the pixel values and what they mean in the CT images produced with the NWC SAF 2013 version. A sample CT image produced with the 2013 version of NWC SAF is given in Figure 3.

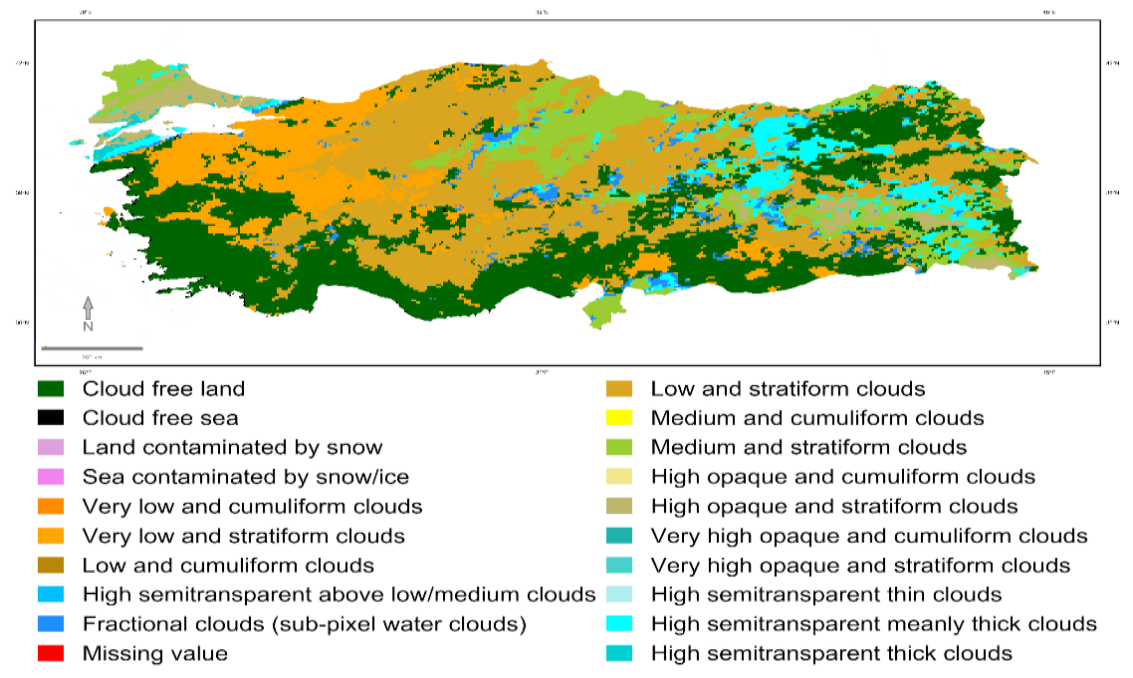

FiguRE 3. A Sample image that we produced using NWC SAF CT data.

${ }^{4}$ https://www.nwcsaf.org/ct_description 
TABLE 4. Meaning of pixel values in NWC SAF CT images.

\begin{tabular}{|c|c|}
\hline Pixel Value & CT (Cloud Types) \\
\hline 0 & Non-processed \\
\hline 1 & Cloud free land \\
\hline 2 & Cloud free sea \\
\hline 3 & Land contaminated by snow \\
\hline 4 & Sea contaminated by snow/ice \\
\hline 5 & Very low and cumuliform clouds \\
\hline 6 & Very low and stratiform clouds \\
\hline 7 & Low and cumuliform clouds \\
\hline 8 & Low and stratiform clouds \\
\hline 9 & Medium and cumuliform clouds \\
\hline 10 & Medium and stratiform clouds \\
\hline 11 & High opaque and cumuliform clouds \\
\hline 12 & High opaque and stratiform clouds \\
\hline 13 & Very high opaque and cumuliform clouds \\
\hline 14 & Very high opaque and stratiform clouds \\
\hline 15 & High semi-transparent thin clouds \\
\hline 16 & High semi-transparent meanly thick clouds \\
\hline 17 & High semi-transparent thick clouds \\
\hline 18 & High semi-transparent above low or medium clouds \\
\hline 19 & Fractional clouds (sub-pixel water clouds) \\
\hline 20 & Undefined (undefined by CMa) \\
\hline
\end{tabular}

\subsubsection{All Sky Camera Data}

DAG-MAM (Meteorological and Astronomical Monitoring) System currently has two all-sky cameras (ASC). These cameras are OMEA and EUDA models from Alcor-System ${ }^{5}$. ASCs monitor the whole sky over the observatory during day and night time (Figure 4). With these cameras, night sky quality and all-night events (Rise and set of Milkyway, meteors/meteoroids, satellite passes etc.) are recorded. The OMEA ASC has a colour sensor from ZWO ASI ${ }^{6}$, with a size of $4150 \times 2800$ pixels. The EUDA ASC has a CCD chip (G2-8300) from Moravian Ints. ${ }^{7}$, with 4600 $\mathrm{x} 3550$ pixels and focal ratio of $\mathrm{f} / 2.8$. The EUDA ASC also has a filter wheel with five standard filters (Light, Red, Green, Blue and Neutral). These two instruments provide an all-sky image in about one-minute temporal resolution.

\footnotetext{
${ }^{5}$ http://www.alcor-system.com/new/AllSky/Omea_camera.html

${ }^{6}$ https://astronomy-imaging-camera.com/

${ }^{7}$ https://www.gxccd.com/?cat=0\&lang $=409$
} 

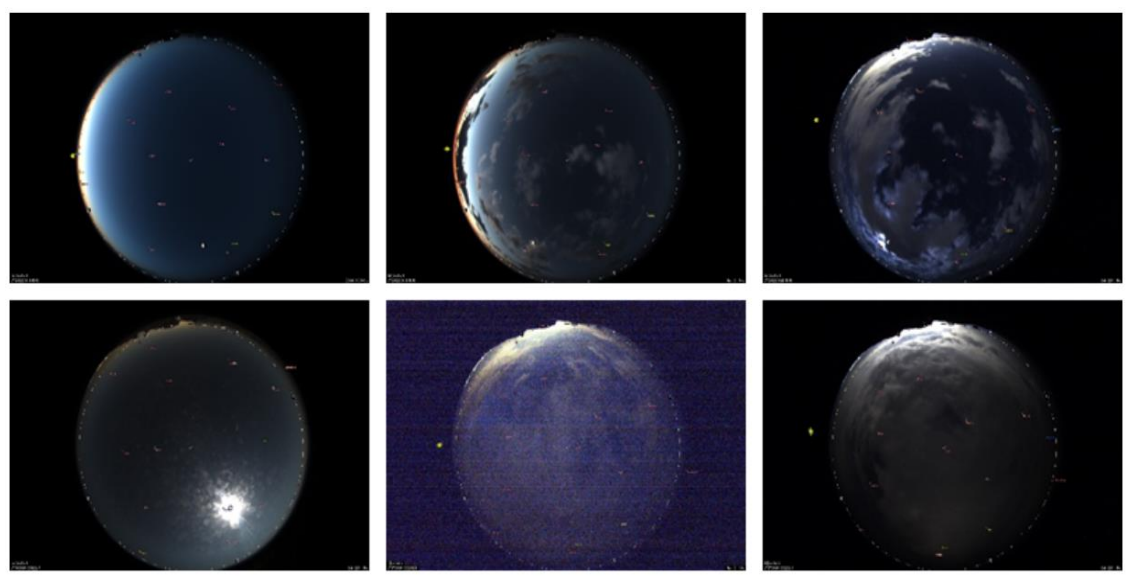

FIGURE 4. ASC images from DAG site.

\subsection{Method}

NWC SAF CMa and CT products used in the study are produced by the TSMS and transmitted to Astrophysics Research and Application Centre (ATASAM) via the internet (i.e. ftp). This data spatially covers Turkey and its surroundings (e.g. Europe, North Africa and the Middle East) with a 15-minute temporal resolution and presented to users in HDF5 (sometimes NetCDF) file format. The CMa and CT data were prepared with the same properties, via Python scripts developed in this study, and then produced in geotiff file format for Turkey (35-43 north latitude and 25-46 east longitude) with about $5 \mathrm{~km}$ (0.045 degrees) spatial pixel resolution, in the geographic coordinate system, geographic latitude/longitude projection, and datum and ellipsoid of WGS84 (World Geodetic System 1984). Afterwards, one-pixel value corresponding to the DAG site from the geotiff images with coordinates of Turkey covering June 1, 2019 - March 31, 2020 timespan was analyzed and used to determine the cloudiness criteria. The criteria determined for the astronomical evaluation of CMa and CT products are given in detail below.

In astronomical observations, not all cloud types affect the observations in the same way. For example, it is known that observations can be made in the presence of clouds such as cirrus and cumulus. Therefore, with the aim of determining whether the sky is clear or cloudy for astronomical purposes, CMa and CT products of NWC SAF have been reclassified, considering the cloud classes of the World 
Meteorological Organisation (WMO), which are created based on the shape and the height at which they are located (see Figure 5 and Table 5). During this reclassification process, considering Turkey's latitudes and the altitude of the DAG site, we used the temperate region values for the cloud levels given in Table 5. Eventually, NWC SAF cloud information is reinterpreted for astronomical observations by using the cloud definitions of WMO, and the experiences of astronomers who have made different kinds of observations in different atmospheric conditions.

TABLE 5. WMO cloud types and their approximate heights for different latitudes ${ }^{8}$ regions.

\begin{tabular}{|c|c|c|c|c|}
\hline Level & Genera & Polar region & Temperate region & Tropical region \\
\hline High & $\begin{array}{c}\text { Cirrus } \\
\text { Cirrocumulus } \\
\text { Cirrostratus }\end{array}$ & $3-8 \mathrm{~km}$ & $5-13 \mathrm{~km}$ & $6-18 \mathrm{~km}$ \\
\hline Middle & $\begin{array}{c}\text { Altocumulus } \\
\text { Altostratus } \\
\text { Nimbostratus }\end{array}$ & $2-4 \mathrm{~km}$ & $2-7 \mathrm{~km}$ & $2-8 \mathrm{~km}$ \\
\hline & $\begin{array}{c}\text { Stratus } \\
\text { Stratocumulus } \\
\text { Cumulus } \\
\text { Cumulonimbus }\end{array}$ & $0-2 \mathrm{~km}$ & $0-2 \mathrm{~km}$ & $0-2 \mathrm{~km}$ \\
\hline
\end{tabular}

\footnotetext{
${ }^{8}$ https://cloudatlas.wmo.int/en/useful-concepts.html
} 


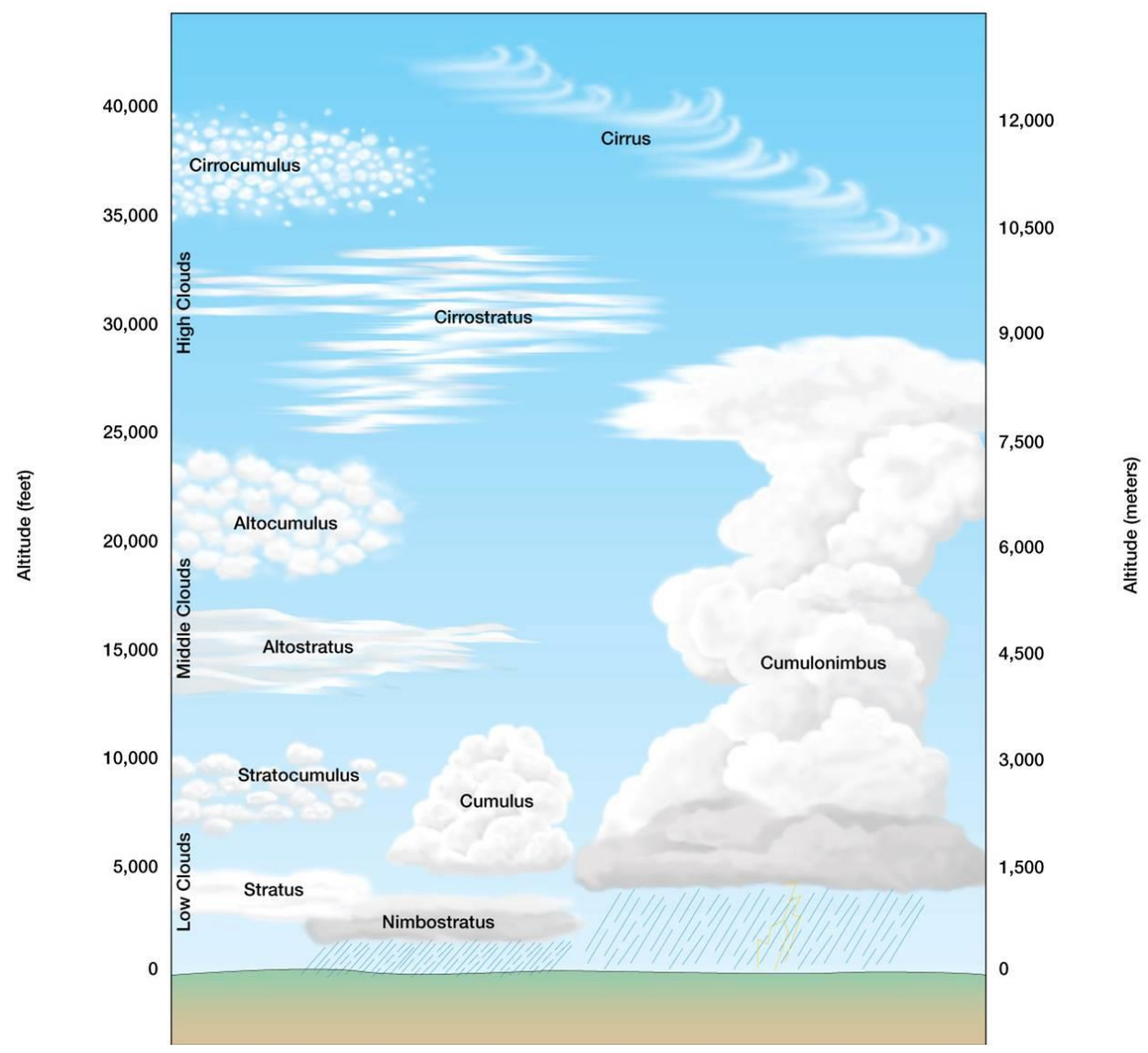

FiguRE 5. WMO cloud classification ${ }^{9}$.

In our study, NWC SAF CMa and CT images with 15-minute temporal resolution from June 1, 2019 to March 31, 2020 were analysed. As a first trial and example, the values of only one pixel ( $\sim 5 \times 5 \mathrm{~km}^{2}$ resolution) of the image at the zenith of the DAG site are used. Combining the analysis of the amount and types of clouds formed over the DAG site, WMO cloud classes, ASC images and the experience of experts on astronomical observations; NWC SAF CMa and CT data were reclassified according to astronomical observation types (photometric, spectroscopic, and unavailable), and renamed as ACMa and ACT (A stands for astronomical).

\footnotetext{
${ }^{9}$ Image Credit: https://puppytales.com.au/wp-content/uploads/2015/04/cloudform2.jpg
} 
Since NWC SAF CMa values are simple (Table 3), ACMa values are obtained with a simple definition and classification. When the pixel value in NWC SAF CMa data is " 0 " (Non-processed) and " 5 " (Undefined), it is not processed $(\mathrm{NaN})$; when the pixel value is "1" (Cloud-free) and "4" (Snow / Ice_contaminated), it is defined as clear (cloudless); when the pixel value is " 2 " (Cloud_contaminated), it is defined as partly cloudy; and when the pixel value is " 3 " (Cloud filled), it is defined as cloudy. If a pixel in NWC SAF cloud data is detected as snow or ice, it should be known that the pixel is cloudless [15].

Unfortunately, NWC SAF CT values are not as simple as CMa values are. Thus, to define ACT values from NWC SAF CT values, several additional parameters are taken into account, such as WMO cloud classes, astronomical observations experts' opinions, and ground-based ASC images (Figure 4). The details of the astronomical reclassification of the NWC SAF cloud types (CT) are given in Table 6 and explained below:

- The cloud types of 5, 7, 9, 11 and 13 are defined as "cloudless" as they are type of cumulus and not visible over the DAG site $(3170 \mathrm{~m})$.

- The cloud types of 6, 8, 10,12 and 14 are defined as "cloudy" as they are opaque and/or stratus type, which completely covers the sky and are opaque, and occur at different levels over the DAG.

- The cloud types of 15,16 and 17 are defined as "partly cloudy" as they are high level semi-transparent types, forming over the DAG site and partially covering the sky.

- The cloud type of 18 is defined as "cloudy" even though they are high-level cirrus type clouds, as there are low and medium level clouds below them.

- The cloud type of 19 is defined as "partly cloudy" as it means there are clouds at some parts of the pixel.

TABLE 6. We gather CMa and CT pixel values into four groups, where we assign new standardised values, and name these new values as ACMa (Astronomical Cloud Mask), ACT (Astronomical Cloud Type), NaN: Not a Number.

\begin{tabular}{|c|c|c|c|c|}
\hline & $\begin{array}{c}\text { CMa Pixel } \\
\text { Value }\end{array}$ & $\begin{array}{c}\text { ACMa } \\
\text { Value }\end{array}$ & CT Pixel Value & $\begin{array}{c}\text { ACT } \\
\text { Value }\end{array}$ \\
\hline Non-processed & 0,5 & NaN & 0,20 & NaN \\
\hline Clear & 1,4 & 0 & $1,2,3,4,5,7,9,11,13$ & 0 \\
\hline Partly cloudy & 2 & 1 & $15,16,17,19$ & 1 \\
\hline Cloudy & 3 & 2 & $6,8,10,12,14,18$ & 2 \\
\hline
\end{tabular}




\section{Results And Discussions}

MSG satellites in geostationary orbit record high temporal resolution images with 15 minutes in nominal scanning. Using these images, the software developed by the EUMETSAT NWC SAF unit produces various atmospheric or meteorological data for nowcasting purposes. From these data, cloud mask (CMa) and cloud type (CT) data were used in this study. ATASAM receives these cloud data, which is produced by TSMS, and covers Turkey and its surroundings within a 15-minute resolution, since May 28, 2019. These data were obtained from TSMS via ftp in the H5 file format. In this study, we used the cloud data covering a 10 month-timespan from June 2019 to March 2020. While it is expected that there will be 29280 images (or files) for each cloud product in the 10 month-timespan; due to data losses during the data transferring and processing procedures, we worked on only 27626 images for $\mathrm{CMa}$ and 27618 images for CT data belonging to this period. Using these data sets, the closest pixel values corresponding to the DAG site, the cloudiness of the DAG site was analysed. The results of the analysis are given in Table 7 and Figure 6 for the CMa, and in Table 8 and Figure 7 the CT values.

As it can be seen in Table 7 and Figure 6, the CMa values for DAG are 53.2\% clear (cloud-free and snow/ice contaminated), 38.0\% cloudy (cloud-filled) and $8.7 \%$ partially cloudy (cloud contaminated). Considering that, according to CMa values, only $38.0 \%$ of the days are cloudy for DAG, and that partially cloudy days are suitable for some observations, it can be said that "good nights" for observation are approximately $61.9 \%$ in total. According to these percentages, approximately 186 days for the 10 months ( 300 days) timespan, which corresponds to 226 days in a year, in the DAG site can be labelled as observable days. Nevertheless, defining the atmosphere as cloudy or clear, and scheduling astronomical observations using only $\mathrm{CMa}$ information may not be very accurate, since one cannot know whether the clouds detected are circus, transparent or low clouds.

TABLE 7. Distribution of pixel values in NWC SAF CMa images for DAG site.

\begin{tabular}{|c|c|c|c|}
\hline NWC SAF Cloud Mask & Pixel Value & Count & Percent \\
\hline Cloud-free & 1 & 13693 & 49.6 \\
\hline Cloud_filled & 3 & 10486 & 38.0 \\
\hline Cloud_contaminated & 2 & 2401 & 8.7 \\
\hline Snow/Ice_contaminated & 4 & 1022 & 3.6 \\
\hline Non-processed & 0 & 24 & 0.1 \\
\hline Total & & $\mathbf{2 7 6 2 6}$ & $\mathbf{1 0 0}$ \\
\hline
\end{tabular}




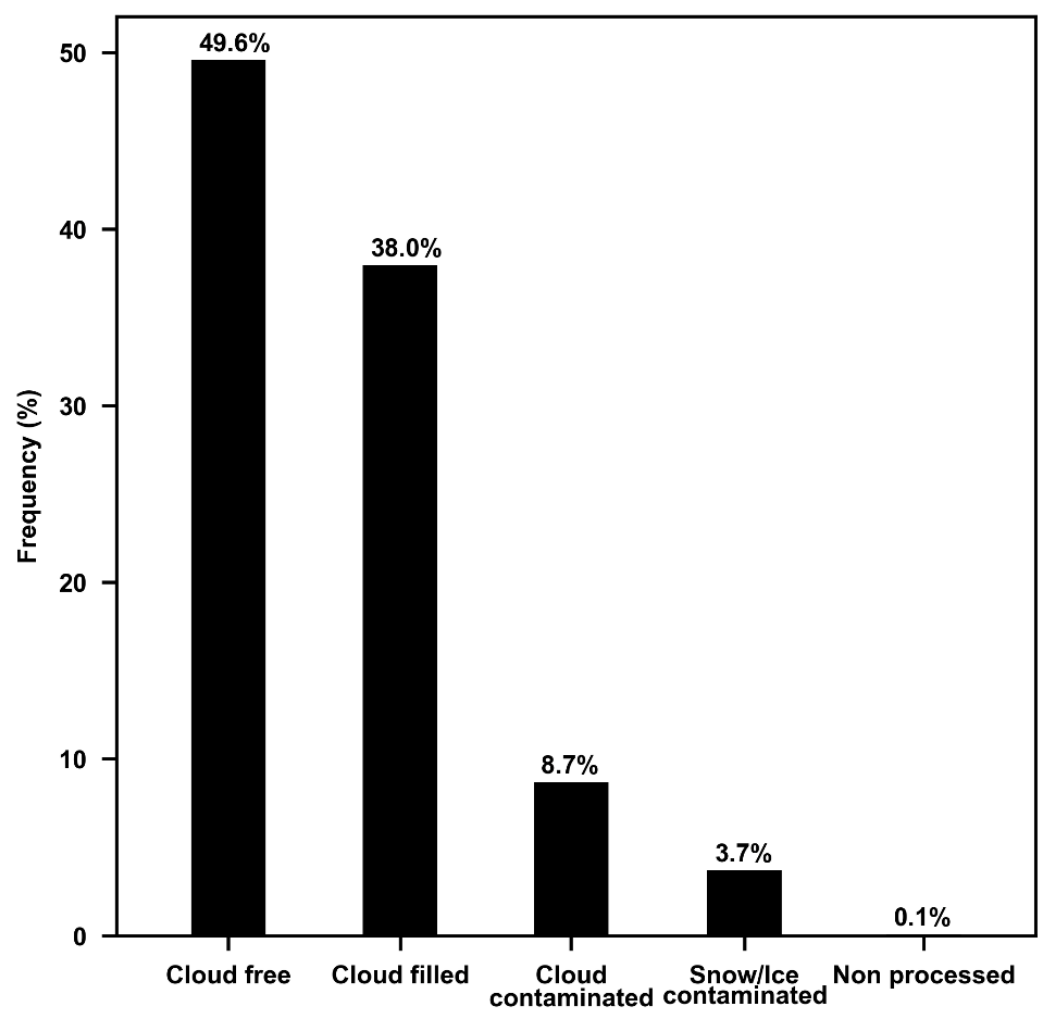

FigURE 6. Distribution of the pixel values in NWC SAF CMa images for DAG site.

In Table 8 and Figure 7, the distribution of NWC SAF CT values determined from images for the 10 month-timespan for DAG is given. NWC SAF CT product provides information in 21 different categorical values, of which 15 are different cloud classes (Table 4). We found that approximately 50\% of our data set do not contain clouds (cloud free land), while 10 different cloud types are observed in the rest. The most common cloud types seen on DAG site are stratiform and high level semi-transparent clouds. On the other hand, it is remarkable that five different cumulus form cloud types are not seen on the DAG site in the aforementioned timespan. While the sum of the cloud-free, snow covered and transparent cloud values from Table 8 is about $68 \%$; the stratus type and opaque cloud values are approximately $31 \%$. Hence, based on these percentages, approximately 204 days are determined as observable days for the 10-month ( 300 days) timespan at the DAG site, which corresponds to approximately 248 days in a year. 
TABLE 8. Distribution of pixel values in NWC SAF CT images for DAG site.

\begin{tabular}{|c|c|c|c|}
\hline NWC SAF Cloud Types & Pixel Value & Count & Percent \\
\hline Cloud free land & 1 & 13692 & 49.6 \\
\hline Medium and stratiform clouds & 10 & 2744 & 9.9 \\
\hline Low and stratiform clouds & 8 & 2555 & 9.2 \\
\hline High opaque and stratiform clouds & 12 & 2147 & 7.8 \\
\hline High semi-transparent meanly thick clouds & 16 & 1593 & 5.8 \\
\hline High semi-transparent thick clouds & 17 & 1554 & 5.6 \\
\hline Land contaminated by snow & 3 & 1022 & 3.7 \\
\hline High semi-transparent above low/medium clouds & 18 & 866 & 3.1 \\
\hline Fractional clouds (sub-pixel water clouds) & 19 & 637 & 2.3 \\
\hline High semi-transparent thin clouds & 15 & 414 & 1.5 \\
\hline Very high opaque and stratiform clouds & 14 & 210 & 0.8 \\
\hline Very low and stratiform clouds & 6 & 160 & 0.6 \\
\hline non-processed & 0 & 24 & 0.1 \\
\hline Total & & $\mathbf{2 7 6 1 8}$ & $\mathbf{1 0 0}$ \\
\hline \multicolumn{2}{|r|}{} & &
\end{tabular}

While the number of clear days in a year for DAG is 226 days determined from the NWC SAF CMa product; it is 248 from the NWC SAF CT product. On the other hand, when the ground-based measurements or different cloud data from different satellites are used to determine the number of clear days for an observatory, diverse numbers are found [16]. Considering these discrepancies, we concluded that the use of CMa and CT products together will yield more accurate results to determine the number of astronomically clear days for an observatory with high accuracy. For this purpose, here we define the "Atmospheric Clearness Index" (ACI) parameter, based on CMa and CT products. In addition to these satellite products, we also used the images from ASCs which are installed at the DAG site and recording the entire sky at a wide angle. The ASC images were visually examined and used as an auxiliary data set to decide whether observations could be performed in corresponding NWC SAF CT cloud types. With an ASC, it can be easily detected whether the sky is clear or cloudy, hence the usage of these data will both confirm and support the accuracy of our results based on the new index, ACI. Thus, with this study, a new parameter to determine observable nights for DAG was developed, and the number of observable nights for the DAG site was determined depending on ACI values for the first time. 


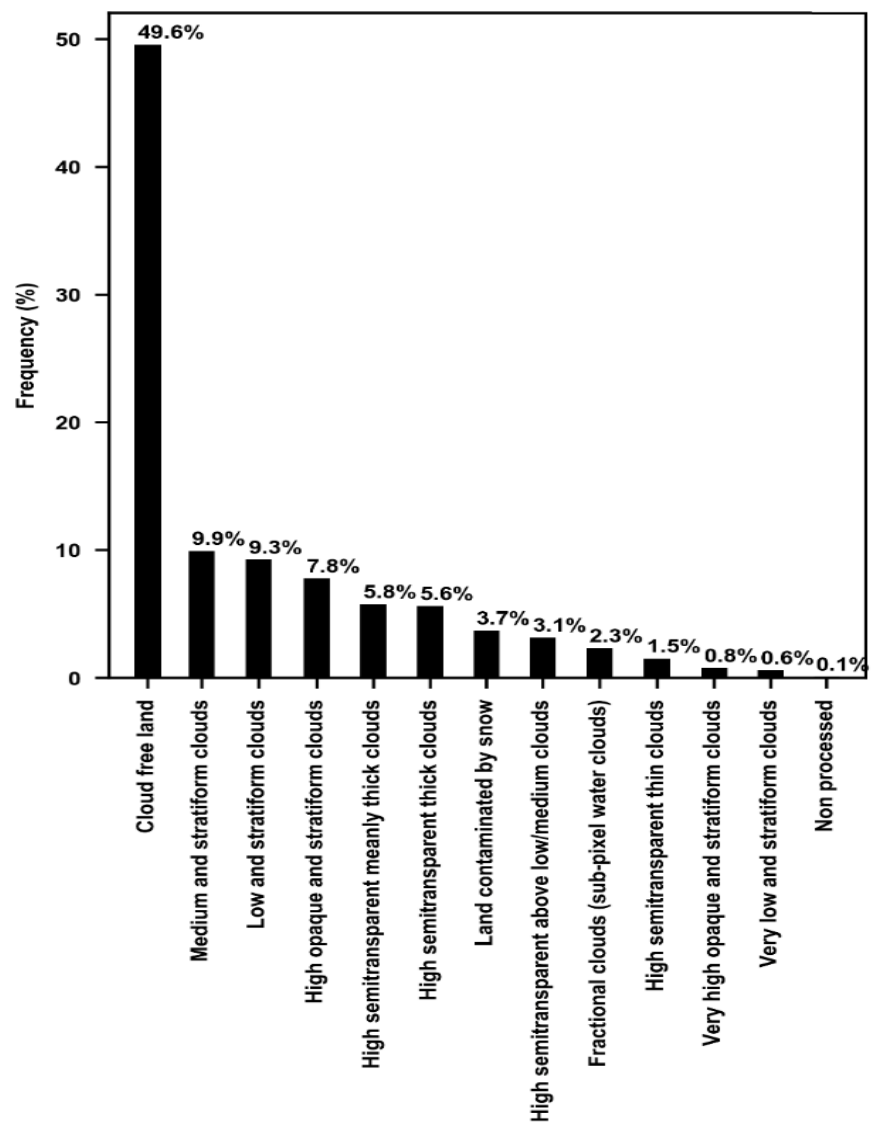

FIGURE 7. Distribution of the pixel values in NWC SAF CT images for DAG site.

Astronomical observations are basically divided into two as spectroscopic (observation of light received from celestial bodies in wavelength resolution) and photometric (observation of light received from celestial bodies in time resolution). In cases where the sky is completely clear (i.e. completely cloudless), firstly, photometric observations are performed (spectroscopic observations can also be made). When the sky is covered partially by clouds, photometric observations are avoided and only spectroscopic observations can be performed. In cases where the sky is completely cloudy, there is no observation opportunity. Therefore, the ACI parameter we define from NWC SAF cloud data, not only indicates whether or not observation can be made, but also which type of observation the weather is suitable for. 
The ACI values and the Astronomical Observation Type (AOT) defined for the different combinations of ACMa and ACT, which are defined based on CMa and $\mathrm{CT}$, are given in Table 9. As the ACI is eventually a parameter based on CMa and CT, it is important to remember the basics about these products. The NWC SAF CT product algorithm first classifies the clouds detected in the CMa product [15]. Therefore, CMa and CT products are expected to be highly compatible with each other. In cases where the CMa product does not give a cloud value, it is expected that there will not be a cloud type in the CT product. Even though this is the case, taking into account the possibility that the $\mathrm{CT}$ algorithm could produce the wrong cloud type value, all combinations of ACT and ACMa were considered, instead of denoting ACT as " 0 " where ACMa is " 0 ".

TABLE 9. We define ACI (Astronomical Clearness Index) based on a combination of ACMa and ACT (see Table 6). ACI takes values of 0,1 or 2 , meaning none, one or two types of astronomical observation (AOT) can be conducted, respectively.

\begin{tabular}{|c|c|c|c|}
\hline ACMA & ACT & ACI & AOT \\
\hline 0 & 0 & 2 & Photometric \\
\hline 0 & 1 & 1 & Spectroscopic \\
\hline 0 & 2 & 0 & Unavailable \\
\hline 1 & 0 & 2 & Photometric \\
\hline 1 & 1 & 1 & Spectroscopic \\
\hline 1 & 2 & 0 & Unavailable \\
\hline 2 & 0 & 2 & Photometric \\
\hline 2 & 1 & 1 & Spectroscopic \\
\hline 2 & 2 & 0 & Unavailable \\
\hline
\end{tabular}

The ACI value being " 2 " means that the cloudiness values of the sky are suitable for quality astronomical observation, for both spectroscopic and photometric techniques. If the ACI value is " 1 ", it means that the sky is partly cloudy and/or only the type of clouds that would not affect spectroscopic observations are present, therefore some astronomical observations can be conducted. The ACI value of "0" means that the sky is too cloudy for any type of astronomical observation. 


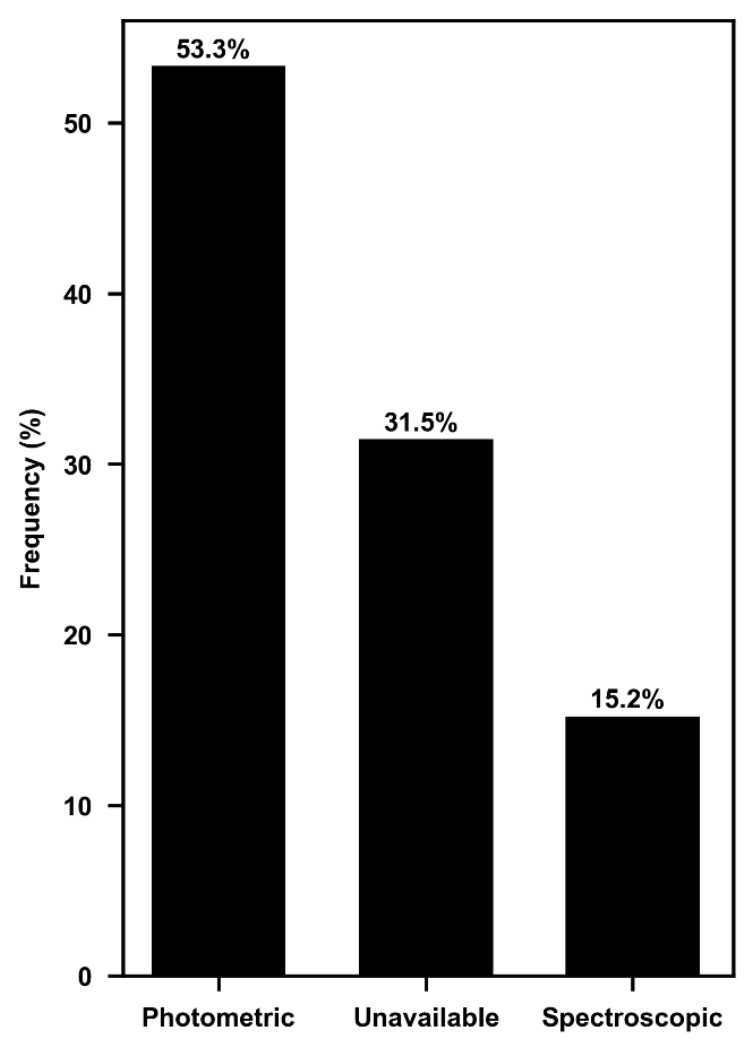

FIGURE 8. Distribution of ACI values for DAG site.

In Figure 8, the distribution of the ACI values produced from the whole data set (10 Months) for DAG is given. From this distribution, it can be seen that about 159 days (53\%) are suitable for both photometric and spectroscopic observations, about 45 days (15\%) is only suitable for spectroscopic observations, and about 93 days (31\%) are not suitable for any type of astronomical observation. Based on these percentages, 204 days ( $\sim 7$ months) are observable days for the 10-month ( 200 days) timespan at the DAG site. Observability is binary as the cloudiness is; however, quality of observations is not, which depends strongly on the type and coverage of the clouds as well as the wavelength of observation [17, 18]. Furthermore, the reader should consider that this result is obtained only by cloudiness, and taking other atmospheric effects such as wind, humidity, temperature and precipitable water vapour into account, observable days will naturally decrease. 


\section{Conclusions}

Knowing the atmospheric conditions instantly and for the very near future (hours, days) is very important for ground-based astronomical observatories. Nowadays, with the usage of remote sensing methods via meteorological satellites, atmosphere monitoring has become easier and more accurate. Two of many products of satellite imagery are CMa and CT cloud products of NWC SAF, which are used in our study. Since the use of any meteorological cloud product alone is not very accurate for astronomical purposes, here we defined a new parameter (ACI); based on two cloud products (CMa and CT), the ASC images taken at the DAG site and WMO cloud definitions and classifications. The first application of the newly developed ACI parameter was performed for the DAG site. According to ACI values calculated, for the 10-month ( 300 days) timespan in the DAG site, approximately 204 days (7 months) are suitable for both photometric and spectroscopic observations, while any observations cannot be performed on 93 days.

\section{Authors Contribution Statement}

Kazım Kaba: He contributed to the determination of the study subject, planning, execution, determination of the method, preparation of the material, conducting the analysis, evaluation of the results and making the study into an article.

Cahit Yeşilyaprak: He contributed to the determination of the study subject, planning, execution, determination of the method, conducting the analysis, evaluation of the results and making the study into an article.

Onur Şatır: He contributed to the preparation of the material, conducting the analysis, evaluating the results and making the study into an article.

\section{Declaration of Competing Interest}

The authors declare that there is no conflict of interests regarding the publication of this paper.

\section{Acknowledgement}

This research is supported by ATASAM and DAG Project (2011K120230) by means of infrastructure and personnel, and also the author Kazım Kaba is supported by TÜBİTAK via $2218(2018 / 2)$ program. 
This publication was created using the TUBITAK-2218-National Postdoctoral Research Fellowship Programme (Project No: 2218-2018/2). However, all responsibility for the publication belongs to the owner of the publication. Financial support received from TUBITAK does not mean that the content of the publication is approved by TUBITAK in a scientific sense.

\section{REFERENCES}

[1] McInnes, B., Walker, M.F., Astronomical site testing in the Canary Islands, Publications of the Astronomical Society of the Pacific, 86 (512) (1974), 529-544. https://doi.org/10.1086/129641

[2] Calisse, P.G., Ashley, M.C.B., Burton, M.G., Phillips, M.A., Storey, J.W.V., Radford, S.J.E., Peterson, J.B., Submillimeter site testing at Dome C, Antarctica, Publications of the Astronomical Society of Australia, 21 (3) (2004), 256-263. https://doi.org/10.1071/AS03018

[3] Schöck, M., Els, S., Riddle, R., Skidmore, W., Travouillon, T., Blum, R., Bustos, E., Chanan, G., Djorgovski, S. G., Gillett, P., Gregory, B., Nelson, J., Otárola, A., Seguel, J., Vasquez, J., Walker, A., Walker, D., Wang, L., Thirty meter telescope site testing I: overview, Publications of the Astronomical Society of the Pacific, 121 (878) (2009), 384395. https://doi.org/10.1086/599287

[4] Vernin, J., Muñoz-Tuñón, C., Sarazin, M., Vazquez Ramió, H., Varela, A.M., Trinquet, H., Delgado, J.M., Jiménez Fuensalida, J., Reyes, M., Benhida, A., Benkhaldoun, Z., Garcia Lambas, D., Hach, Y., Lazrek, M., Lombardi, G., Navarrete, J., Recabarren, P., Renzi, V., Sabil, M., Vrech, R., European extremely large telescope site characterization I: overview, Publications of the Astronomical Society of the Pacific, 123 (909) (2011), 1334-1346. https://doi.org/10.1086/662995

[5] Hidayat, T., Mahasena, P., Dermawan, B., Hadi, T. W., Premadi, P. W., Herdiwijaya, D., Clear sky fraction above Indonesia: an analysis for astronomical site selection, Monthly Notices of the Royal Astronomical Society, 427 (3) (2012), 1903-1917. https://doi.org/10.1111/j.1365-2966.2012.22000.x

[6] Koc-San, D., San, B. T., Bakış, V., Helvacı, M., Eker, Z., Multi-Criteria Decision Analysis integrated with GIS and remote sensing for astronomical observatory site selection in Antalya province, Turkey, Advances in Space Research, 52 (1) (2013), 3951. https://doi.org/10.1016/j.asr.2013.03.001

[7] Aksaker, N., Yerli, S. K., Erdoğan, M. A., Erdi, E., Kaba, K., Ak, T., Aslan, Z., Bakış, V., Demircan, O., Evren, S., Keskin, V., Küçük, İ., Özdemir, T., Özışı, T., Selam, S. O., Astronomical site selection for Turkey using GIS techniques, Experimental Astronomy, 39 (3) (2015), 547-566. https://doi.org/10.1007/s10686-015-9458-x

[8] Falvey, M., Rojo, P.M., Application of a regional model to astronomical site testing in western Antarctica, Theoretical and Applied Climatology, 125 (3-4) (2016), 841-862. https://doi.org/10.1007/s00704-016-1794-x

[9] Hellemeier, J.A., Yang, R., Sarazin, M., Hickson, P., Weather at selected astronomical sites - an overview of five atmospheric parameters, Monthly Notices of the Royal Astronomical Society, 482 (4) (2019), 4941-4950. https://doi.org/10.1093/mnras/sty2982 
[10] Aksaker, N., Yerli, S.K., Erdoğan, M.A., Kurt, Z., Kaba, K., Bayazit, M., Yeşilyaprak, C., Global site selection for astronomy, Monthly Notices of the Royal Astronomical Society, 493 (1) (2020), 1204-1216. https://doi.org/10.1093/mnras/staa201

[11] Badescu, V., Dumitrescu, A., CMSAF products Cloud Fraction Coverage and Cloud Type used for solar global irradiance estimation, Meteorology and Atmospheric Physics, 128 (4) (2016), 525-535. https://doi.org/10.1007/s00703-015-0424-y

[12] Chernokulsky, A., Esau, I., Cloud cover and cloud types in the Eurasian Arctic in 19362012, International Journal of Climatology, 39 (15) (2019), 5771-5790. https://doi.org/10.1002/joc.6187

[13] Kotarba, A.Z., Chacewicz, S., Żmudzka, E., Night sky photometry over Warsaw (Poland) evaluated simultaneously with surface-based and satellite-based cloud observations, Journal of Quantitative Spectroscopy and Radiative Transfer, 235 (2019), 95-107. https://doi.org/10.1016/j.jqsrt.2019.06.024

[14] Toy S, Kantor N. Evaluation of human thermal comfort ranges in urban climate of winter cities on the example of Erzurum city. Environmental Science and Pollution Research, 24 (2) (2017), 1811-1820. https://doi.org/10.1007/s11356-016-7902-8

[15] Derrien, M., Le Gléau, H., MSG/SEVIRI cloud mask and type from SAFNWC, International Journal of Remote Sensing, 26 (21) (2005), 4707-4732. https://doi.org/10.1080/01431160500166128

[16] Yüzlükoğlu F., Erzurum ve çevresinin astronomi gözlemleri açısından atmosferik özellikleri. M.Sc. Thesis, Atatürk University, Erzurum, Turkey, 2017.

[17] Marks, R. D., Astronomical seeing from the summits of the Antarctic plateau, Astronomy \& Astrophysics, 385 (1) (2002), 328-336. https://doi.org/10.1051/0004-6361:20020132

[18] Seghouani, N., Boer, M., \& Mimouni, J., National Aures Observatory: A new multimessenger facility, Journal of Physics: Conference Series, 1269 (1) (2019), 012001. 